\title{
Efecto de facilitación de los cognados en el cerebro bilingüe
}

\author{
M. ${ }^{a}$ Ángeles Palomar-García \\ mpalomar@uji.es \\ ELISENDA BUEICHEKÚ BOHABONAY \\ bueichek@uji.es \\ ANA SANJUÁN TOMÁS \\ asanjuan@uji.es \\ NoElia VEntura-CAmpos \\ venturan@uji.es \\ CÉSAR ÁVILA \\ avila@uji.es
}

\section{Resumen}

Introducción: El objetivo de la presente investigación de resonancia magnética funcional fue estudiar las diferencias neurales y conductuales entre bilingües y monolingües durante una tarea de denominación de palabras cognadas y no cognadas a partir de imágenes. Metodología: La muestra estaba formada por 33 estudiantes universitarios diestros: 16 bilingües de castellano-catalán, cuya L1 era el castellano, y 17 monolingües de castellano. Se realizó una tarea con un diseño asociado a eventos lento en RMf que consistió en la denominación en castellano (L1) a partir de imágenes, donde la mitad de las imágenes se denominaban con palabras cognadas y la otra mitad con palabras no cognadas. Resultados: Los resultados conductuales obtenidos mostraron un claro efecto de cognación y una interacción entre el estado de cognación y el efecto del grupo, por el que los bilingües denominaban más rápido palabras cognadas que no cognadas. Los resultados neurales mostraron diferencias globales entre bilingües y monolingües en áreas posteriores, pero estas no fueron específicas para palabras cognadas o no cognadas. También se observaron diferencias en el grupo de los bilingües, observándose mayor activación en el córtex cingulado anterior (ACC) cuando la condición de palabras no cognadas fue comparada con las palabras cognadas. Conclusión: Tal y como postula la hipótesis de la interferencia, el grupo de los bilingües presenta una mayor participación del córtex cingulado anterior durante el procesamiento de palabras no cognadas debido a la activación en paralelo del léxico de L1 y de L2.

Palabras claves: cognados, bilingüismo, córtex cingulado anterior (ACC), neuroimagen, resonancia magnética funcional (RMf).

\section{Abstract}

Introduction: The aim of the present fMRI research was to study the neural and behavioral differences between bilinguals and monolinguals during picture naming task with cognate and 
non-cognate words. Method: The sample consisted of 33 right-handed university students: 16 Spanish - Catalan bilinguals and 17 Spanish monolinguals. For this study, a slow event-related fMRI task was used which consisted in a picture naming task performed in Spanish (L1). The pictures were manipulated for cognate status, thus half of them were named with cognate words and the other with half non-cognate words. Result: The behavioral results obtained showed a main effect of the cognate status and an interaction between the cognate status and the effect of group by which the bilingual denominated faster cognates words than non-cognate words. The neural results showed overall differences between bilinguals and monolinguals in posterior areas, but these were not specific for cognate or non-cognate words. Differences within the bilingual group were observed, revealing an increased activation in the anterior cingulate cortex (ACC) when the non-cognate condition was compared to the cognate condition. Conclusion: As the interference hypothesis postulates, bilinguals have a parallel activation of L1 and L2 lexicons that could be related to the increased activation observed in the anterior cingulate cortex (ACC) while processing non cognate words.

Keywords: cognates, bilingualism, anterior cingulate cortex (ACC), neuroimaging, functional magnetic resonance (fMRI).

\section{Introducción}

El término bilingüe se refiere a una persona con la capacidad de utilizar más de un idioma con fluidez (Abutalebi y cols. 2008). Este proceso es especialmente relevante en nuestra sociedad donde castellano y catalán se alternan continuamente. Una persona bilingüe tiene una lengua materna o primera lengua (denominada L1) en la que muestra una alta proficiencia y una segunda lengua aprendida (denominada L2) en la que puede tener distintos niveles de proficiencia. En el contexto del bilingüismo castellano-catalán es frecuente encontrar bilingües con alta proficiencia en L2 ya que han aprendido ambos idiomas simultáneamente.

Los cognados son palabras que comparten el mismo significado en los dos idiomas y son fonológicamente similares (ej., oreja en castellano y orella en catalán) y los no cognados son palabras que también comparten el mismo significado pero fonológicamente son distintos (ej., cama en castellano y llit en catalán). Las palabras cognadas y no cognadas solamente tienen sentido en el contexto del bilingüismo (Costa, Caramazza y Sebastián-Gallés, 2000).

La investigación previa sobre estudios conductuales en producción del lenguaje ha mostrado que cuando se compara a los bilingües con los monolingües en diferentes tareas de producción verbal, como tareas de denominación, de lectura y de fluidez verbal, los bilingües presentan un mayor tiempo de respuesta cuando denominan en su segunda lengua (L2) como cuando denominan en su primera lengua (L1). Esta desventaja se ha observado para ambos tipos de palabras cognadas y no cognadas (Gollan y cols. 2005, 2008; Ivanova y cols. 2008; Rosselli y cols. 2000). Costa y cols. (2000) realizaron un estudio en el que mostraron el efecto de facilitación para las palabras cognadas en los bilingües, y encontraron diferencias significativas del efecto del cognado para el grupo de los bilingües, y observó diferencias significativas del efecto del cognado para el grupo de los bilingües, obteniendo menores tiempos de reacción para las palabras cognadas que para las no cognadas. Este efecto de facilitación de los cognados puede ser encontrado para personas con diferentes niveles de proficiencia, no solamente en su L2 sino incluso para su lengua dominante L1 (Christoffels y cols., 2003, 2006). 
Los estudios de neuroimagen han mostrado una mayor activación en áreas lingüísticas del hemisferio izquierdo ( $\mathrm{HI}$ ) para los bilingües en comparación con los monolingües cuando realizan tareas de denominación, lectura en voz alta o tareas de decisión léxica. Parker-Jones y cols. (2012) encontraron incremento de activación en cinco áreas del HI: giro precentral dorsal, pars triangularis, pars opercularis, giro temporal superior y el plano temporal, para los bilingües comparado con los monolingües durante tareas de denominación y lectura en su L1, pero no durante el procesamiento semántico. Rodríguez-Fornells y cols. (2002) también encontraron un incremento de activación en áreas del $\mathrm{HI}$ como el córtex frontal inferior, el plano temporal y el lóbulo parietal inferior, en una tarea de decisión léxica en su L1 cuando comparaba bilingües con monolingües.

Kroll y cols. (2008) proponen que estas diferencias entre grupos lingüísticos son debidas a que los bilingües deben activar en paralelo los dos idiomas cuando hablan en su L1, produciéndose una interferencia mutua entre la producción de L1 y L2. Ambos idiomas pueden ser activados, pero los bilingües desarrollan la habilidad de seleccionar a los candidatos del idioma objetivo. Es, por tanto, la activación de un mayor número de competidores léxicos junto con la menor frecuencia de uso de las palabras por el hecho de hablar dos idiomas lo que produce la mayor interferencia (Parker-Jones y cols., 2012).

El objetivo principal de nuestro estudio fue investigar las diferencias conductuales y neurales entre bilingües y monolingües durante una tarea de denominación en L1 con palabras cognadas y no cognadas. En concreto nos planteamos conocer las diferencias globales en denominación y la influencia de los cognados y no cognados en la respuesta neural al realizar una tarea de denominación a partir de imágenes. Las hipótesis que nos planteamos para este estudio fueron: a) los bilingües deberían tener tiempos de denominación (TD) más lentos que los monolingües y presentar un incremento de activación en áreas del hemisferio izquierdo; $b$ ) los bilingües deberían denominar las palabras no cognadas más lentamente que las palabras cognadas y esta diferencia debería verse reflejada en el patrón de activación cerebral cuando las palabras no cognadas están siendo procesadas.

\section{Metodología}

\section{Participantes}

La muestra estaba formada por 33 estudiantes de la Universitat Jaume I de Castellón: 16 bilingües castellano-catalán y 17 monolingües. Todos los participantes fueron asignados al grupo de bilingües o monolingües después de que ellos completaran un cuestionario sobre el uso de los idiomas. Este cuestionario permite obtener la edad de adquisición (todos los participantes bilingües aprendieron ambos idiomas antes de los tres años) y el nivel de proficiencia para cada participante mediante una escala de 4 puntos ( $1=$ pobre nivel de proficiencia, $4=$ alto nivel de proficiencia), todos los participantes bilingües mostraron una alta proficiencia en ambos idiomas.

A pesar de la temprana y continua exposición a ambos idiomas, los participantes mostraban una preferencia por uno de sus idiomas. Con ello, la L1 de todos los participantes era el castellano.

Todos los participantes eran diestros y fueron informados de la naturaleza del estudio dando su consentimiento para participar en él. La participación en este estudio fue monetariamente recompensada. 
Tarea

La tarea utilizada en este estudio fue una tarea de denominación. Consistió en la presentación de 64 imágenes de objetos familiares seleccionados de Strijkers, Costa y Thierry (2010) que se presentaban durante 1,5 segundos. La mitad de las imágenes se denominaban con palabras cognadas y la otra mitad con palabras no cognadas. Casi todas las palabras cognadas (27 de 32) compartían, por lo menos, la primera sílaba entera con su correspondiente traducción, y todos ellos comparten al menos el primer fonema.

Las imágenes fueron presentadas en la pantalla del ordenador y los participantes fueron instruidos en dar una respuesta en voz alta para cada imagen y en denominarlas con la máxima rapidez.

El paradigma de resonancia fue aplicado mediante un diseño asociado a eventos lento. Cada ensayo empezaba con un punto de fijación blanco sobre un fondo negro que permanecía en la pantalla durante 1 segundo y mantenía la atención del participante en el centro de la pantalla, inmediatamente después aparecía la imagen que duraba 1,5 segundos. Después la pantalla permanecía en negro durante 13,5 segundos hasta el siguiente ensayo, cada ensayo duraba 16 segundos. Durante los ensayos experimentales, los participantes debían responder en voz alta y durante los ensayos línea de base ( «+» y la pantalla en negro), debían ver pasivamente la pantalla.

El orden de las imágenes fue aleatorio para cada participante y la sesión duraba aproximadamente 17 minutos.

La tarea fue programada y presentada utilizando el Software Presentation (Neurobehavioral System, INC; Albany, CA), e implementado mediante el sistema operativo Microsoft XP. Los estímulos visuales fueron presentados dentro del escáner usando unas gafas compatibles con la resonancia (Resonance Technologies, EE. UU.) y las respuestas verbales de los participantes fueron grabadas mediante un micrófono compatible que cancela el ruido del escáner (FOMRI, Optoacoustic, Israel).

Parámetros de adquisición de las imágenes de Resonancia Magnética funcional (RMf)

Las imágenes se obtuvieron mediante un escáner Siemens Avanto (Erlangen, Alemania) de 1,5 T. Los participantes fueron colocados en una posición supina en el escáner de resonancia magnética. Para reducir los artefactos producidos por el movimiento se utilizaron cojines para fijar sus cabezas.

Antes de la secuencia funcional de resonancia magnética, para cada participante se adquirió un volumen estructural usando una secuencia MPRAGE de alta resolución potenciada en T1 (TE $=3.8 \mathrm{~ms}, \mathrm{TR}=2200 \mathrm{~ms}$, FOV $=25.6 \times 25.6 \mathrm{~cm}$, tamaño vóxel $=1 \times 1 \times 1 \mathrm{~mm}$ ). A continuación, se adquirió una secuencia gradiente-echo T2* echo-planar de 148 volúmenes usada para las imágenes funcionales $(\mathrm{TE}=55 \mathrm{~ms}$, $\mathrm{TR}=3000 \mathrm{~ms}$, matriz $=64 \times 64$, tamaño vóxel $=3.5 \times 3.5 \times 3.8$ ). Se adquirieron 33 cortes axiales intercalados de manera ascendente, paralelos a la comisura anterior / comisura posterior cubriendo todo el cerebro.

\section{Análisis conductuales}

El tiempo de denominación (TD) y el juicio de las respuestas (acierto, error, omisión) fueron obtenidos manualmente usando el programa Praat (http://www.fon.hum.uva.nl/praat/). 
Los tiempos de denominación (TD) fueron basados solamente en las respuestas correctas, y las respuestas que superaban 3 desviaciones típicas fueron excluidas de los análisis.

Una vez obtenidos los TD se analizaron con el programa Statistical Package for the Social Sciences (SPSS v.19.0). Se llevaron a cabo pruebas paramétricas (ANOVA de $2 \times 2$ ).

\section{Análisis de las imágenes}

El procesamiento de las imágenes y los análisis estadísticos fueron realizados usando el programa SPM8 (Statistical Parametric Mapping 8; Wellcome Trust Centre for Neuroimaging, London, UK. http://www.fil.ion.ucl.ac.uk/spm).

El Preprocesado de las imágenes funcionales incluía corrección donde todos los volúmenes fueron temporalmente alineados («slice-timing»), corrección por movimiento, normalización espacial de la imágenes funcionales a un espacio esterotáxico estandarizado -coordenadas del Instituto Neurológico de Montreal (MNI) de $3 \mathrm{~mm}^{3}$-. Finalmente sobre los datos funcionales se aplicó un suavizado espacial con una función gaussiana mediante full width half maximum (FWHM) de $8 \mathrm{~mm}^{3}$.

El análisis estadístico fue realizado sobre los datos individuales y grupales utilizando el Modelo Lineal General (Friston y cols. 1995). Para cada participante, los contrastes de cada condición versus la línea de base fueron calculados (cognados > línea de base, no-cognados $>$ línea de base). Los datos de cada participante se modelaron mediante un diseño asociado a eventos lento con la función de la respuesta hemodinámica y su derivada temporal. Los parámetros obtenidos de la corrección de movimiento, los TD y los errores se introdujeron para cada participante como covariable en el análisis de efectos fijos.

Los efectos de interés grupal (bilingües/monolingües) de cada uno de los contrastes (cognado y no cognado) obtenidos para cada participante en los análisis de efectos fijos, fueron estudiados mediante una prueba t para una muestra. Las diferencias entre grupos (bilingües y monolingües) en cada contraste fueron obtenidas mediante una prueba t para dos muestras. Para todos los resultados se aplicó un umbral family-wise error (FWE) $p<0.05$ corregido por múltiples comparaciones a nivel de clúster mediante la simulación de Montecarlo usando el programa Alphasim de la herramienta REST (http://www.restfmri.net), con un umbral de $p<0.005$ no corregido a nivel de vóxel y un criterio de tamaño de clúster de 45 vóxeles.

\section{Resultados}

\section{Resultados conductuales}

El análisis de los datos conductuales (tabla 1) muestra la media y la desviación típica para cada una de las condiciones experimentales. Este análisis fue llevado a cabo mediante un análisis de varianza (ANOVA) del grupo (bilingües vs. monolingües) y el estado del cognado (cognadas vs. no cognadas) mostrando una tendencia a ser significativo el efecto de grupo ( $F$ $(1,31)=3,185 p=0,08)$ y diferencias significativas para el efecto de cognado $(F(1,31)=5,518$ $p<0,05)$. También se encontró un efecto significativo para la interacción entre el estado del cognado y el grupo $(F(1,31)=8,089 p<0,01)$. Como se esperaba, el TD para los bilingües fue más lento (1243 ms) que para los monolingües $(1185 \mathrm{~ms})$ y dentro del grupo de los bilingües más rápido para las palabras cognadas (1219 ms) que para las no cognadas (1267 ms), no existiendo diferencias entre ambas en monolingües. Por lo tanto, fue obtenido el efecto de facilitación para las palabras cognadas. 
Tabla 1

\begin{tabular}{lcccc}
\hline & \multicolumn{2}{c}{ GRUPO BILINGÜES } & \multicolumn{2}{c}{ GRUPO MONOLINGÜES } \\
\hline & TD & DT & TR & DT \\
COGNADAS & 1219 & 200 & 1113 & 195 \\
NO COGNADAS & 1267 & 217 & 1124 & 194 \\
DIFERENCIA & -48 & & -11 & \\
\hline
\end{tabular}

$\mathrm{TD}=$ Media del tiempo de denominación (milisegundos). DT = Desviación típica.

\section{Resultados de RMf}

La figura 1 muestra las regiones donde se observaron diferencias significativas cuando realizamos los efectos principales de la tarea, es decir, cuando juntamos a los dos grupos (bilingües y monolingües) y las dos condiciones (cognados y no cognados), encontrándose áreas relacionadas con la denominación, como el área de Broca y Wernicke.

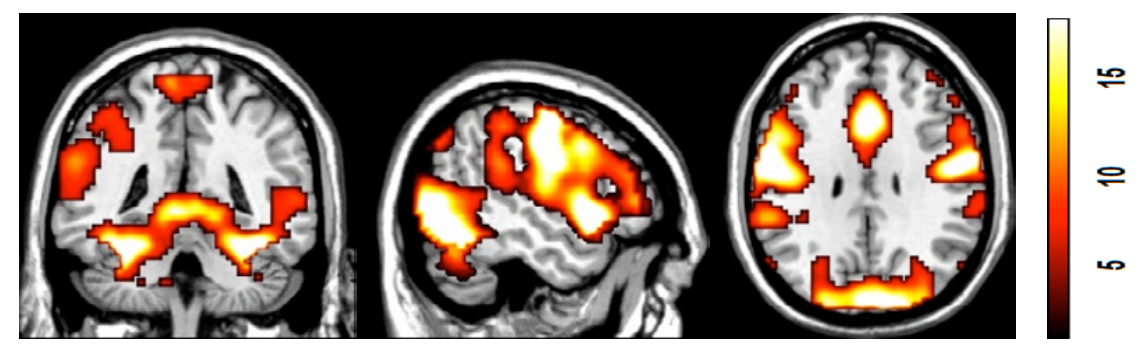

La parte derecha de la imagen representa la parte derecha del cerebro. Mapa de activación obtenido con umbrales $p<0,005$ corregido por múltiples comparaciones a nivel de clúster $p<0.05$.

Figura 1. Activaciones obtenidas para los efectos principales de la tarea

Cuando realizamos comparaciones entre grupos se observan diferencias significativas entre ambos grupos (véanse tabla 3 y figura 2). El grupo bilingüe mostró mayores activaciones que el grupo monolingüe cuando comparamos las dos condiciones (cognados y no cognados) en giro angular bilateral y giro cingulado posterior izquierdo. Por otro lado, el grupo de los monolingües, presentó una mayor activación en giro temporal medio e inferior bilateral y giro occipital medio bilateral. Otras activaciones aparecen en la tabla 3 para el grupo de los bilingües y monolingües, respectivamente. 
(a)
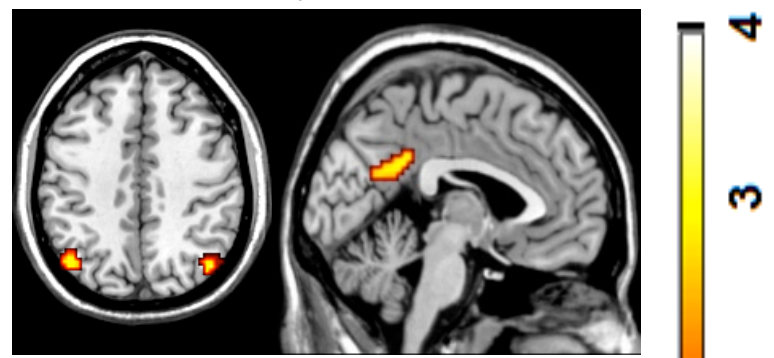

(b)

N

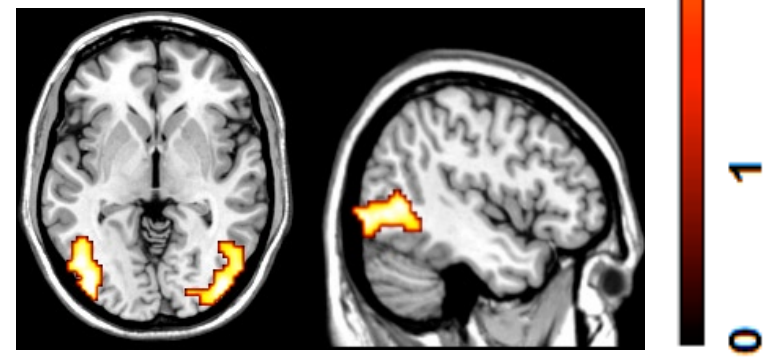

La parte derecha de la imagen representa la parte derecha del cerebro. Mapa de activación obtenido con umbrales $p<0,005$ corregido por múltiples comparaciones a nivel de clúster $p<0.05$.

Figura 2. Activaciones cerebrales cuando comparamos bilingües frente a monolingües (a) y monolingües frente a bilingües $(b)$ en todas las condiciones de la tarea

Tabla 3

Regiones que mostraron activación cerebral cuando comparamos ambos grupos durante todas las condiciones de la tarea (cognadas y no cognadas)

\begin{tabular}{|c|c|c|c|c|}
\hline \multirow[t]{2}{*}{ Áreas cerebrales } & \multirow[t]{2}{*}{ BA } & \multicolumn{3}{|c|}{ Tarea } \\
\hline & & Clúster & Puntuación Z & Coordenadas MNI \\
\hline \multicolumn{5}{|l|}{ Bilingües > Monolingües } \\
\hline Giro angular derecho & 19,39 & 54 & 3.36 & $45,-73,40$ \\
\hline Giro angular izquierdo & 39 & 50 & 3.11 & $-42,-67,40$ \\
\hline Giro cingulado posterior izquierdo & 31 & 64 & 3.08 & $-3,-46,31$ \\
\hline \multicolumn{5}{|l|}{ Monolingües > Bilingües } \\
\hline Giro occipital medio izquierdo & 18,37 & 300 & 4.99 & $-42,-85,-5$ \\
\hline Giro temporal medio izquierdo & 39 & & 3.94 & $-45,-61,4$ \\
\hline Giro occipital medio derecho & 19,39 & 323 & 4.43 & $42,-85,-2$ \\
\hline Giro temporal medio derecho & 37 & & 4.19 & $54,-73,1$ \\
\hline Giro temporal inferior derecho & 37 & & 3.79 & $48,-64,-8$ \\
\hline Giro fusiforme derecho & 19 & & 3.31 & $36,-70,-8$ \\
\hline Cisura calcarina derecha & 17 & & 3.07 & $18,-88,4$ \\
\hline
\end{tabular}

$\mathrm{BA}=$ Áreas de Brodmann. 
Las diferencias entre grupo para cada condición produjeron resultados muy parecidos a las comparaciones grupales. Quizá este patrón de resultados obedezca a las diferencias en tiempo de denominación entre grupos.

Se realizaron análisis adicionales para comparar el procesamiento de cognados y no cognados en cada grupo. La tabla 4 y la figura 3 muestran las regiones donde se observaron diferencias significativas en la respuesta BOLD en el grupo de los bilingües. En el contraste cognados > no cognados solo se encontró una diferencia en el giro temporal medio derecho. Por otro lado, el contraste inverso, no cognados > cognados produjo una mayor respuesta en el giro cingulado anterior y giro angular bilateral.

(a)

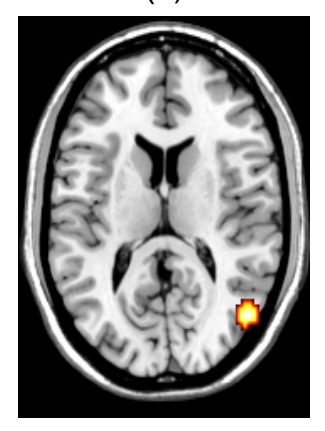

(b)

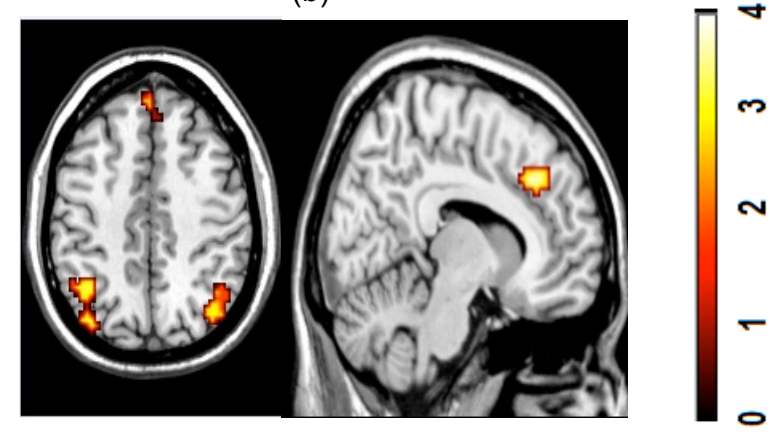

La parte derecha de la imagen representa la parte derecha del cerebro. Mapa de activación obtenido con umbrales $p<0,005$ corregido por múltiples comparaciones a nivel de clúster $p<0.05$.

Figura 3. Activaciones cerebrales en el grupo de los bilingües cuando comparamos las palabras cognadas frente a las no cognadas (a) y comparación de las no cognadas frente a las cognadas $(b)$

Tabla 4

Regiones que mostraron activación cerebral para el grupo bilingüe cuando comparamos las condiciones de cognadas y no cognadas

\begin{tabular}{lcccc}
\hline Áreas cerebrales & BA & \multicolumn{3}{c}{ Tarea } \\
\hline $\begin{array}{l}\text { Cognadas> No cognadas } \\
\text { Giro temporal medio derecho }\end{array}$ & 39 & 49 & 4.05 & $48,-70,13$ \\
& & & & \\
No cognadas > Cognadas & & & & \\
Giro cingulado anterior derecho & 32 & 117 & 3.71 & $9,29,37$ \\
Giro frontal medial derecho & 9,8 & & 3.43 & $3,32,37$ \\
Giro angular izquierdo & 39,40 & 73 & 3.66 & $-39,-76,43$ \\
Giro angular derecho & 40 & 54 & 3.43 & $42,-70,49$ \\
\hline
\end{tabular}




\section{Discusión}

El paradigma de RMf aplicado en este trabajó estudió la respuesta cerebral de un grupo de bilingües (castellano-catalán) y uno de monolingües (castellano) durante una tarea de denominación en L1 a partir de imágenes. La mitad de las imágenes correspondían a palabras cognadas y la otra mitad eran no cognadas. Los bilingües denominaron más lento que los monolingües especialmente las palabras no cognadas. La tarea no confirmó resultados previos que mostraron diferencias entre grupos en el lóbulo frontal inferior izquierdo (Parker Jones y cols. 2012; Rodríguez-Fornells y cols., 2002) pero sí que se encontró mayor activación en el giro angular bilateral (véase Rodríguez-Fornells y cols., 2002). Las comparaciones intragrupo en los bilingües mostró mayor activación en el córtex cingulado anterior (ACC) y el giro angular bilateral cuando comparamos la condición de no cognadas con cognadas.

Ttal como muestran los resultados conductuales, la ejecución de la tarea mostró una tendencia a que el tiempo de denominación fuera más lento para el grupo de los bilingües en relación al grupo de monolingües. Este efecto es debido a las mayores dificultades de acceso léxico que se observan en bilingües en cualquiera de los dos idiomas (Kroll y cols. 2008). Dentro del grupo de los bilingües se observa un efecto de facilitación para las palabras cognadas, denominándose más rápido que las palabras no cognadas. Este efecto ya había sido obtenido en investigaciones previas (Costa y cols. 2000; Christoffels y cols. 2003, 2006). El efecto de facilitación para las palabras cognadas se produce por la existencia de interacción entre los niveles léxicos y subléxicos de representación de las palabras (Costa y cols., 2009). Cuando se presenta una imagen de una palabra cognada, tanto el nodo léxico objetivo (oreja) como su traducción (orella) comparten segmentos fonológicos (/o/, /r/, /e/, /a/). Por lo tanto, el nodo léxico objetivo (oreja) recibe activación de ambos sistemas semánticos y de ambos segmentos fonológicos. Por otro lado, cuando el nodo léxico objetivo es una palabra no cognada (cama - llit (catalán)) recibe menor activación del segmento fonológico, ya que no hay ningún solapamiento fonológico entre la palabra objetivo y su traducción. Este efecto de facilitación puede servir como un índice de hasta qué punto el idioma no objetivo se activa y por lo tanto influye en el procesamiento del idioma objetivo.

Los resultados también mostraron que los bilingües activaban en mayor medida el giro cingulado posterior izquierdo y en el giro angular bilateral. Este incremento de activación puede ser debido al peor acceso léxico que presentan los bilingües debido a la menor frecuencia de uso. En cambio en el grupo de los monolingües se encontraron mayores activaciones en áreas occipitales que pueden ser relacionadas con el mejor acceso léxico que presentan.

Este incremento de activación para el grupo de los bilingües cuando denominan en su primera lengua (castellano) en la región parietal posterior izquierda, ya ha sido reportado en la literatura previa (Rodríguez-Fornells y cols. 2002) y se ha relacionado con la respuesta motora. Abutalebi y Green (2008) proponen que esta área está relacionada con la selección del idioma objetivo.

Cuando realizamos los análisis intragrupo, comparando la condición de no cognadas frente a cognadas para los bilingües, observamos un incremento de activación en el giro cingulado anterior, este resultado es lo esperado, ya que es un área que se ha relacionado con la detección del conflicto de idiomas (Abutalebi y Green, 2007, 2008). Estudios previos con tareas donde se alternan dos idiomas han mostrado activación del ACC y se ha relacionado con el control y selección del idioma (Abutalebi y cols. 2012; Van Heuven y cols., 2008). Estudios recientes consideran que esta región evalúa la demanda o necesidad de control cognitivo cuando aparece un conflicto o interferencia en el procesamiento de dos estímulos o respuestas (Carter y cols., 2000; Botvinick y cols., 1999). 
El incremento de activación para el grupo de los bilingües se puede interpretar como que necesitan un mayor reclutamiento de esta área durante el procesamiento de palabras no cognadas debido a la activación en paralelo del léxico de L1 y L2 tal y como postula la hipótesis de la interferencia (Kroll y cols. 2008). Por lo tanto, la activación de Acc está directamente relacionada con el grado de respuesta de conflicto presente en una determinada tarea cognitiva.

En resumen, en el presente estudio se ha demostrado cómo los bilingües muestran una desventaja en el acceso al léxico cuando se comparan con los monolingües, incluso cuando la tarea es realizada en su primer idioma o idioma dominante (L1). Además estos datos sugieren que la competición es acentuada en los bilingües y para poder denominar las imágenes es necesario que inhiban el idioma no objetivo y seleccionen el idioma objetivo.

\section{Bibliografía}

Abutalebi, J. \& Green, D. (2007). Bilingual language production: The neurocognition of language representation and control. Journal of Neurolinguistic, 20(3), 242-275.

Abutalebi, J. \& Green, D. (2008). Control mechanisms in bilingual language production: Neural evidence from language switching studies. Language and Cognitive Processes, 23(4), 557582.

Abutalebi, J., Annoni, J-M., Zimine, I., Pegna, A. J., Segier, M. L., Lee-Jahnke, H., Lazeyras, F., Cappa, S. F. \& Khateb, A. (2008). Language Control and Lexical Competition in Bilinguals: An Event-Related fMRI Study. Cerebral Cortex, 18(7), 1496-1505.

Abutalebi, J., Della-Rosa, P. A., Green, D., Hernandez, M., Scifo, P., Keim, R., Cappa, S. F. \& Costa, A. (2012). Bilingualism Tunes the Anterior Cingulate Cortex for Conflict Monitoring. Cerebral Cortex, 22(9), 2076-2086.

Botvinick, M., Nystrom, L. E., Fissell, K., Carter, C. S. \& Cohen, J. D. (1999). Conflict monitoring versus selection-for-action in the anterior cingulate cortex. Nature, 402, 179-181.

Carter, C. S., Macdonald, A. M., Botvinick, M., Ross, L. L., Stenger, V. A., Noll, D. \& Cohen, J. D. (2000). Parsing executive processes: Strategic vs. evaluative functions of the anterior cingulate cortex. Proceedings of the National Academy of Sciences, EE. UU., 97(4), 19441948.

Christoffels, I. K., De Groot, A. M. B. \& Waldorp, L. J. (2003). Basic skills in a complex task: a graphical model relating memory and lexical retrieval to simultaneous interpreting. Bilingualism: Language and Cognition, 6(3), 201-211.

Christoffels, I. K., De Groot, A. M. B. \& Kroll, J. F. (2006). Memory and language skills in simultaneous interpreters: The role of expertise and language proficiency. Journal of. Memory and Language, 54(3), 324-345.

Costa, A., Caramazza, A. \& Sebastián-Gallés, N. (2000). The cognate facilitation effect: Implications for models of lexical access. Journal of Experimental Psychology: Learning, Memory and Cognition, 26(5), 1283-1296.

Costa, A., Strijkers, K., Martin, C. \& Thierry, G. (2009). The time course of word retrieval revealed by event-related brain potentials during overt speech. Proc Natl Acad Sci EE. UU., 106(50), 21442-6.

Friston, K. J., Holmes, A. P., Poline, J-B., Grasby, P. J., Williams, S. C. R., Frackowiak, R. S. J. \& Turner, R. (1995). Analysis of fMRI Time-Series Revisited. Neuroimage, 2(1), 45-53.

Gollan, T. H., Montoya, R. I., Fennema-Notestine, C. \& Morris, S. K. (2005). Bilingualism affects picture naming but not picture classification. Memory \& Cognition, 33(7), 1220-1234. 
Gollan, T. H., Montoya, R. I., Cera, C. \& Sandoval, T. C. (2008). More use almost always a means a smaller frequency effect: Aging, bilingualism, and the weaker links hypothesis. Journal of Memory and Language, 58(3), 787-814.

Ivanova, I. \& Costa, A. (2008). Does bilingualism hamper lexical access in speech production? Acta Psychologica, 127, 277-288.

Kroll, J. F., Bobb, S. C., Misra, M. \& Guo, T. (2008). Language selection in bilingual speech: Evidence for inhibitory processes. Acta psychologica, 128(3), 416-430.

Parker-Jones, O., Green, D. W., Grogan, A., Pliatsikas, C., Filippopolitis, K., Ali, N., Lee, H. L, Ramsden, S., Gazarian, K., Seghier, M. L. \& Price, C. J. (2012). Where, when and why brain activation differs for bilinguals and monolinguals during picture naming and reading aloud. Cereb Cortex, 22(4), 892-902.

Rodríguez-Fornells, A., Rotte, M., Heinze, H. J., Nösselt, T. \& Münte, T. F. (2002). Brain potential and functional MRI evidence for how to handle two languages with one brain. Nature, 415, 1026-1029.

Rosselli, M., Ardila, A., Araujo, K., Weekes, V. A., Caracciolo, V., Padilla, M. \& Ostrosky-Solí, F. (2000). Verbal Fluency and Repetition Skills in Healthy Older Spanish-English Bilinguals. Applied Neuropsychology, 7(1), 17-24.

Strijkers, K., Costa, A. \& Thierry, G. (2010). Tracking Lexical Access in Speech Production: Electrophysiological Correlates of Word Frecuency and Cognate Effects. Cerebral Cortex, 20(4), 912-928.

Van Heuven W. J., Schriefers, H., Dijkstra, T. \& Hagoort, P. (2008). Language Conflict in the Bilingual Brain. Cerebral Cortex, 18(11), 2706. 